\title{
Influence of the cellulose substrate on the electrochemical properties of paper-based polypyrrole electrode materials
}

\author{
Henrik Olsson • Daniel O. Carlsson • \\ Gustav Nyström • Martin Sjödin • Leif Nyholm • \\ Maria Strømme
}

Received: 4 January 2012/Accepted: 15 March 2012/Published online: 3 April 2012

(c) The Author(s) 2012. This article is published with open access at Springerlink.com

\begin{abstract}
The influence of the cellulose substrate on the electrochemical performance of supercapacitor electrode materials made of polypyrrole (PPy) and cellulose is investigated. Composites were synthesized by chemical polymerization of pyrrole on dispersed fibers of cellulose from Cladophora algae and dispersed wood cellulosebased commercial filter papers, respectively, as well as on Cladophora cellulose and filter paper sheets. The resulting composites, which were characterized using scanning electron microscopy, cyclic voltammetry, and elemental analysis, were found to exhibit specific charge capacities proportional to the PPy content of the composites. The highest specific capacity (i.e., $171 \mathrm{C} / \mathrm{g}$ composite or 274 $\mathrm{C} / \mathrm{g}$ PPy) was obtained for composites made from dispersed Cladophora cellulose fibers. The higher specific capacities for the Cladophora cellulose composites can be explained by the fact that the Cladophora cellulose fibers were significantly thinner than the wood cellulose fibers. While the PPy was mainly situated on the surface of the Cladophora cellulose fibers, a significant part of the PPy was found to be present within the wood fibers of the filter paper-based
\end{abstract}

Electronic supplementary material The online version of this article (doi:10.1007/s10853-012-6418-y) contains supplementary material, which is available to authorized users.

H. Olsson · D. O. Carlsson · G. Nyström · M. Sjödin ·

M. Strømme $(\square)$

Nanotechnology and Functional Materials,

The Ångström Laboratory, Uppsala University,

P. O. Box 534, 75121 Uppsala, Sweden

e-mail: Maria.Stromme@angstrom.uu.se

L. Nyholm $(\bowtie)$

Department of Materials Chemistry, The Ångström Laboratory,

Uppsala University, P. O. Box 538, 75121 Uppsala, Sweden

e-mail: Leif.Nyholm@mkem.uu.se composites. The latter can be ascribed to a higher accessibility of the aqueous pyrrole solution to the wood-based fibers as compared to the highly crystalline algae based cellulose fibers. The present results clearly show that the choice of the cellulose substrate is important when designing electrode materials for inexpensive, flexible and environmentally friendly paper-based energy storage devices.

\section{Introduction}

There is currently a high demand for lightweight, inexpensive, flexible, and environmentally friendly energy storage devices for a number of applications including new types of portable electronics, propulsion of all-electric or hybrid vehicles as well as for the management of intermittent renewable energy sources [1-6].

As a result of these needs, research is currently carried out to develop both thin flexible lithium-ion batteries [6] and novel current collectors for such devices [7]. Another approach to flexible energy storage involves the utilization of conducting polymers, which offer promising aspects in terms of easy manufacturing, inexpensive raw materials and environmental friendliness $[8,9]$. Since the discovery of the conductive properties of doped polyacetylene [10], a wide variety of polymers with conductive properties have been presented $[11,12]$. One of the most promising candidates is polypyrrole [11] (PPy) which can be polymerized in aqueous solutions, and offers good stability as well as a theoretical charge storage capacity of $418 \mathrm{C} / \mathrm{g}$ assuming a $\mathrm{Cl}^{-}$doping level of $33 \%$ [13]. A commonly used approach for making PPy electrodes is to polymerize the monomer, pyrrole, onto a substrate. This can be done by electro polymerization using electrically conducting substrates 
[14], but also by chemical polymerization onto both electrically conducting [15] and non-conducting substrates [16-18].

Two main problems with conducting polymer based batteries and supercapacitors are their poor cycling stabilities [11, 19], and rate restrictions due to mass transport limitations within thick polymer layers [20, 21]. To overcome the latter problem, we have previously developed a material based on a chemically deposited thin $(\sim 50 \mathrm{~nm})$ layer of PPy coated on a large surface area substrate of dispersed nanofibrous cellulose from the Cladophora algae [22], which could be used in both paper-based supercapacitors [23, 24] and in electrochemically controlled anion exchange procedures [25-27] including DNA extraction [28] and hemodialysis [29, 30]. We have also demonstrated that it is possible to produce a material with similar electrochemical properties using dispersed microfibrillated cellulose from wood as substrate for the chemical polymerization of PPy [31]. In addition, it was recently shown that the PPy-Cladophora cellulose composites exhibited excellent cycling stability, with only $0.7 \%$ loss in capacitance over 4000 cycles, when used as the electrodes in an aqueous symmetric supercapacitor device [32]. It was further shown that no significant loss in capacitance occurred when charging the device to $1.8 \mathrm{~V}$ due to an intrinsic self-protective mechanism which prevented degradation of the PPy [32].

In situ polymerization of conducting polymers on cellulose can be carried out based on three main approaches involving (i) mixing or soaking the cellulose in a monomer solution followed by addition of the oxidant [18], (ii) soaking the cellulose in a solution of the oxidant followed by addition of monomer solution [33] and (iii) soaking of the cellulose in oxidant solution followed by addition of monomer from the vapor phase [34]. To enable the manufacturing of conducting polymer layers on cellulose with varying thickness, layer-by-layer techniques have been proposed based on deposition from solutions containing oligomers [17, 35], vapor polymerization [34, 36] and deposition from polymer solutions in organic solvents [37]. However, to the best of our knowledge, the influence of the cellulose substrate on the electrochemical performance of the composites has not yet been systematically studied. In the present work, we investigate how different cellulose substrate types and composite manufacturing processes affect the electrochemical behavior of the obtained PPycellulose composites. For this purpose, we use composites manufactured from paper sheets as well as from dispersed fibers of Cladophora cellulose and commercial filter paper and discuss the influence of the composite morphology on the electrochemical properties of the materials. We also investigate the possibilities of preparing thicker PPy layers (than the previously obtained $\sim 50 \mathrm{~nm}$ layers) by carrying out the polymerization step several times or with different amounts of the reagents.

\section{Experimental}

Sample preparation

Composites prepared from dispersed Cladophora cellulose

Cladophora sp. algae cellulose was prepared as described elsewhere [38]. Pyrrole ( $>97 \%)$, iron (III) chloride hexahydrate $(>99 \%)$, Tween 80 and sodium chloride $(>99.5 \%)$ (VWR international) were used as received, and deionized water was used throughout the synthesis. Three composites were prepared similarly starting with Cladophora cellulose that was sonicated until dispersed ( $\sim 8 \mathrm{~min})$ in a beaker containing water using a VibraCell sonicator (Sonics, USA). A pyrrole solution containing a few drops of Tween-80 was then added to the cellulose dispersion and the mixture stirred for 1-2 min using a magnetic stirrer. Polymerization was subsequently carried out by adding a solution of $\mathrm{FeCl}_{3}$ to the mixture. The reaction was left to proceed under constant stirring, after which the resulting black slurry was transferred to a Büchner funnel and thoroughly washed with water. After the washing step, the mixture was sonicated for 1 additional minute. As a final step, all water was drained from the funnel and the resulting black paper composite was peeled off and left to dry at room temperature. The first of the three different composites was prepared using $68 \mathrm{ml}$ of $6 \mathrm{mg} / \mathrm{ml}$ cellulose dispersion, $135 \mathrm{ml} 0.4 \mathrm{M}$ pyrrole and $132 \mathrm{ml} 0.3 \mathrm{M} \mathrm{FeCl}_{3}$ solutions, and a polymerization time of $10 \mathrm{~min}$. The second composite was synthesized using double amounts of pyrrole and $\mathrm{FeCl}_{3}$ relative to the amount of cellulose substrate- $106 \mathrm{ml} 0.8 \mathrm{M}$ pyrrole and $100 \mathrm{ml}$ $0.6 \mathrm{M} \mathrm{FeCl}_{3}$-employing $50 \mathrm{ml} 6 \mathrm{mg} / \mathrm{ml}$ cellulose dispersion and a polymerization time of $20 \mathrm{~min}$. The third composite was made using the same relative concentrations as in the first synthesis, but in this case a second subsequent polymerization step was also carried out on the black slurry and the polymerization time was $20 \mathrm{~min}$ in each step. The third composite was thus also based on double reactant amounts compared to the first composite.

\section{Composite prepared from dispersed filter paper}

A composite made from dispersed filter paper (Whatman grade no. 42, UK) was prepared by sonicating $300 \mathrm{mg}$ filter paper in $50 \mathrm{ml}$ of water for $\sim 8 \mathrm{~min}$ until the paper was completely dispersed. After this, $100 \mathrm{ml} 0.4 \mathrm{M}$ pyrrole solution was added to the dispersion and the mixture was 
sonicated for an additional minute. Polymerization was started by adding $100 \mathrm{ml} 0.3 \mathrm{M} \mathrm{FeCl}_{3}$ under constant stirring. After $2 \mathrm{~min}, 100 \mathrm{ml}$ water was added and the polymerization was allowed to continue for an additional $28 \mathrm{~min}$. The product was washed and collected as described in the previous section.

\section{Composites prepared from sheets of dip-coated Cladophora cellulose paper and filter paper}

Four different composites were prepared from paper sheets-both commercial filter paper (Whatman grade no. 42, UK) and paper made from Cladophora cellulose. The Cladophora cellulose paper was made by dispersing Cladophora cellulose in water and filtering the dispersion in a Büchner funnel with applied filter paper. The Cladophora cellulose formed a film that could be peeled off and dried to form a paper sheet. Dip-coated composites were made by soaking the paper sheets in a $3.3 \mathrm{M}$ pyrrole solution and then placing them in a $3.0 \mathrm{M} \mathrm{FeCl}_{3}$ solution for $10 \mathrm{~min}$. After this, the composites were washed with water and left to dry. Two dip-coated composites, made from commercial filter paper and Cladophora cellulose paper, respectively, were produced exactly as described above. The third and fourth dip-coated composites were made with commercial filter paper substrates, and the polymerization procedure was repeated two and three times, respectively, in an attempt to increase the amount of PPy in the composites.

Table 1 summarizes the sample preparation procedures for all composites.

\section{Characterization}

Electrochemical characterization was performed using an Autolab PGSTAT302 N potentiostat (Ecochemie, The Netherlands). Cyclic voltammetry (CV) was performed in $2.0 \mathrm{M} \mathrm{NaCl}$ aqueous electrolyte at scan rates in the range of $5-50 \mathrm{mV} / \mathrm{s}$ utilizing a standard three-electrode setup with a coiled Pt-wire as auxiliary electrode and a $3.0 \mathrm{M}$ $\mathrm{NaCl} \mathrm{Ag/AgCl}$ electrode as reference. The composites, which served as the working electrodes, were cut into small pieces $(\sim 5 \mathrm{mg})$ and contacted with Pt-wire folded into a pocket. All potentials are given versus the $\mathrm{Ag} / \mathrm{AgCl}$ reference electrode.

Scanning electron microscopy (SEM) micrographs were obtained with a LEO1550 field emission SEM instrument (Zeiss, Germany). The composites were dried and mounted on aluminum stubs using a conductive adhesive tape. Additionally, cross-section SEM micrographs were obtained using a cross-section polisher (Jeol, Japan) and a JSM-7401F microscope (Jeol, Japan).

The PPy content of the samples was evaluated by elemental analysis ( $\mathrm{CHN}$-analysis performed by the company Eurofins MikroKemi AB, Uppsala, Sweden).

\section{Results and discussion}

As is seen in Figs. 1 and 2, the SEM micrographs for the composites prepared by dip-coating of paper sheets and from dispersed cellulose fibers exhibited different morphologies. The composite made from dip-coated Cladophora cellulose

Table 1 Sample preparation conditions for the composites presented in this paper

\begin{tabular}{|c|c|c|c|c|c|c|c|}
\hline Sample name & Substrate & Cellulose & $\begin{array}{l}\text { Pyrrole } \\
\text { solution }\end{array}$ & $\begin{array}{l}\mathrm{FeCl}_{3} \\
\text { solution }\end{array}$ & $\begin{array}{l}\text { Number of } \\
\text { polymerizations }\end{array}$ & $\begin{array}{l}\text { Polymerization } \\
\text { time (min) }\end{array}$ & Comments \\
\hline Clad_paper_dip-1 & $\begin{array}{l}\text { Cladophora } \\
\text { cellulose paper } \\
\text { sheet }\end{array}$ & $206 \mathrm{mg}$ & $3.3 \mathrm{M}$ & $3.0 \mathrm{M}$ & 1 & 10 & \\
\hline Filter_paper_dip-1 & Filter paper sheet & $120 \mathrm{mg}$ & $3.3 \mathrm{M}$ & $3.0 \mathrm{M}$ & 1 & 10 & $\begin{array}{l}\text { Dipped in pyrrole solution } \\
\text { and placed in } \mathrm{FeCl}_{3} \\
\text { during polymerization }\end{array}$ \\
\hline Filter_paper_dip-2 & Filter paper sheet & $100 \mathrm{mg}$ & $3.3 \mathrm{M}$ & $3.0 \mathrm{M}$ & 2 & $2 \times 10$ & \\
\hline Filter_paper_dip-3 & Filter paper sheet & $187 \mathrm{mg}$ & $3.3 \mathrm{M}$ & $3.0 \mathrm{M}$ & 3 & $3 \times 10$ & \\
\hline $\begin{array}{l}\text { Filter_paper_fibers- } \\
\quad 1\end{array}$ & $\begin{array}{l}\text { Dispersed filter } \\
\text { paper cellulose }\end{array}$ & $\begin{array}{l}50 \mathrm{ml} \\
6 \mathrm{mg} / \mathrm{ml}\end{array}$ & $\begin{array}{r}100 \mathrm{ml} \\
0.4 \mathrm{M}\end{array}$ & $\begin{array}{l}100 \mathrm{ml} \\
0.3 \mathrm{M}\end{array}$ & 1 & $2+28$ & $\begin{array}{l}100 \mathrm{ml} \text { water added after } \\
2 \mathrm{~min}\end{array}$ \\
\hline Clad_fibers-1 & $\begin{array}{l}\text { Dispersed } \\
\text { Cladophora } \\
\text { cellulose }\end{array}$ & $\begin{array}{l}68 \mathrm{ml} \\
6 \mathrm{mg} / \mathrm{ml}\end{array}$ & $\begin{array}{l}135 \mathrm{ml} \\
0.4 \mathrm{M}\end{array}$ & $\begin{array}{l}132 \mathrm{ml} \\
0.3 \mathrm{M}\end{array}$ & 1 & 10 & \\
\hline Clad_fibers-2 & $\begin{array}{l}\text { Dispersed } \\
\text { Cladophora } \\
\text { cellulose }\end{array}$ & $\begin{array}{l}50 \mathrm{ml} \\
6 \mathrm{mg} / \mathrm{ml}\end{array}$ & $\begin{array}{c}103 \mathrm{ml} \\
0.4 \mathrm{M}\end{array}$ & $\begin{array}{c}100 \mathrm{ml} \\
0.3 \mathrm{M}\end{array}$ & 2 & $2 \times 20$ & $\begin{array}{l}\text { In total twice the reactants } \\
\text { of Clad_fibers-1 }\end{array}$ \\
\hline $\begin{array}{l}\text { Clad_fibers- } \\
\text { 1double }\end{array}$ & $\begin{array}{l}\text { Dispersed } \\
\text { Cladophora } \\
\text { cellulose }\end{array}$ & $\begin{array}{l}50 \mathrm{ml} \\
6 \mathrm{mg} / \mathrm{ml}\end{array}$ & $\begin{array}{c}106 \mathrm{ml} \\
0.8 \mathrm{M}\end{array}$ & $\begin{array}{c}100 \mathrm{ml} \\
0.6 \mathrm{M}\end{array}$ & 1 & 20 & $\begin{array}{l}\text { Twice the reactants of } \\
\text { Clad_fibers-1 }\end{array}$ \\
\hline
\end{tabular}



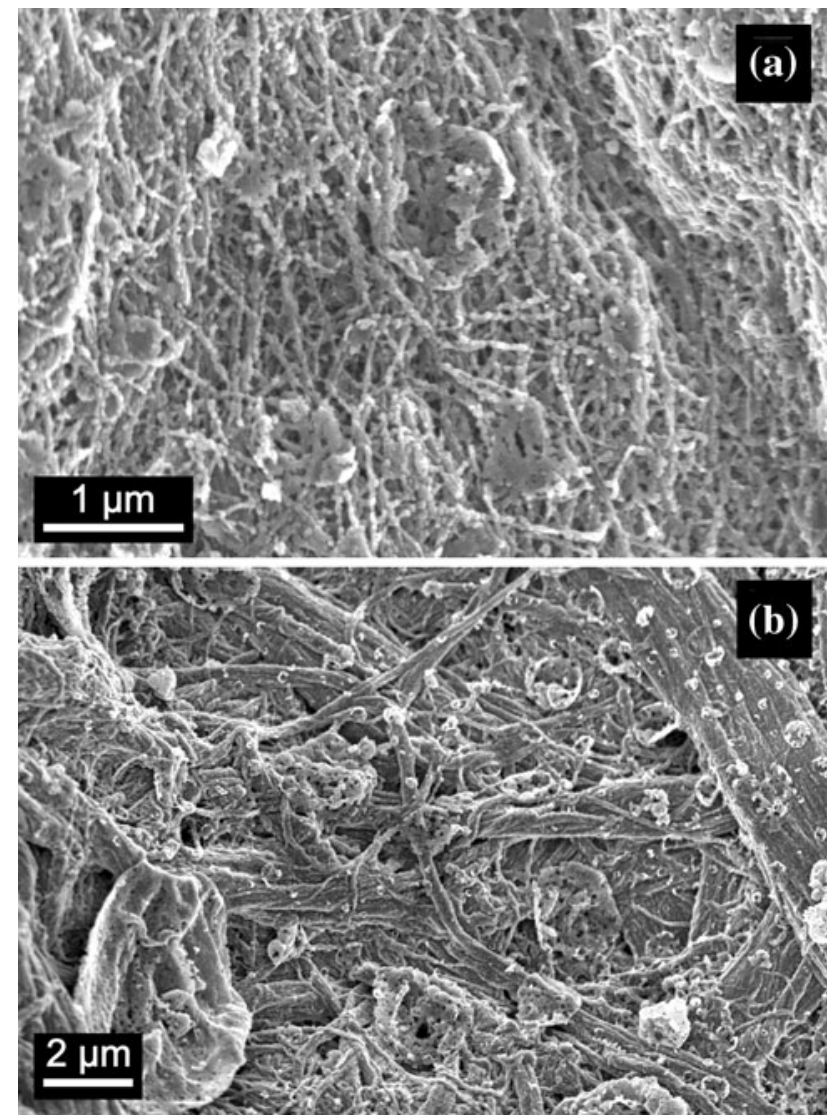

Fig. 1 SEM micrographs depicting a a PPy/paper composite prepared by dip-coating of a Cladophora cellulose paper sheet (Clad_paper_dip-1) and b a PPy/paper composite prepared by dipcoating of a filter paper sheet (Filter_paper_dip-1)

paper sheets (Fig. 1a), exhibited the previously presented characteristic nanosized fibrillar structure [22], but appeared more compact than the composites prepared from dispersed Cladophora cellulose (see Fig. 2). The composites prepared from dip-coated filter paper sheets (Fig. 1b) exhibited a compact structure much like the filter paper itself with larger, micron sized, fibers. A cross-section SEM micrograph of the latter sample (see Fig. 3a), revealed bead like structures, characteristic of PPy, on fibers throughout the sample. Corresponding cross-section SEM micrographs of composites prepared from dispersed Cladophora cellulose (Fig. 3b) revealed a structure very similar to that seen in the surface SEM micrograph (Fig. 2), indicating that the polymerization had taken place on all the dispersed fibers and that this type of composite had a more homogeneous structure than the composites obtained via polymerization performed on paper sheets.

Micrographs obtained for the three different composites prepared from dispersed Cladophora cellulose fibers (Fig. 2), indicate the presence of similar morphologies, although the composite prepared with twice the amount of pyrrole (Clad_fibers-1double) compared to the standard synthesis (Clad_fibers-1) appeared to have slightly more
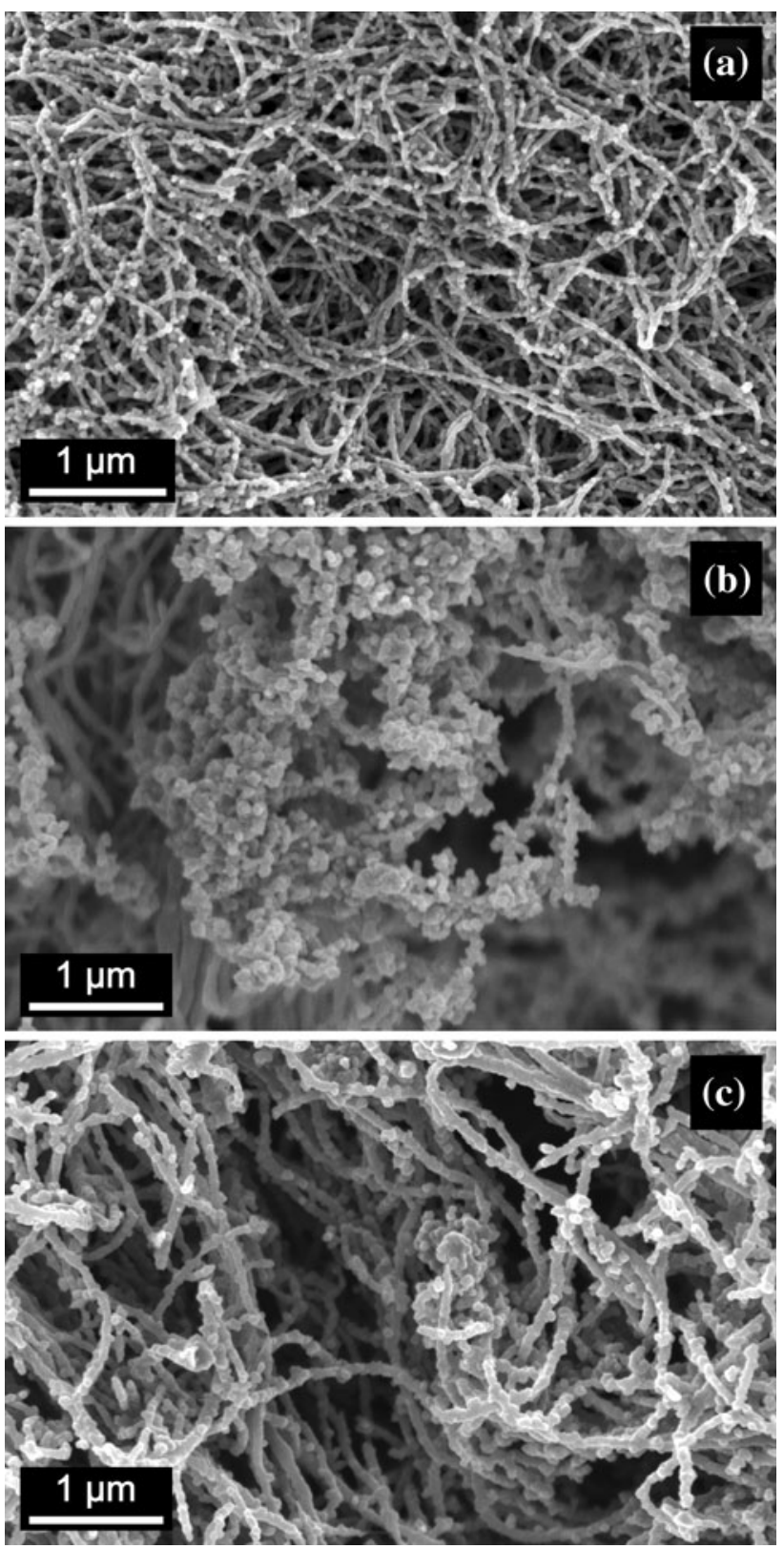

Fig. 2 SEM micrographs of PPy/Cladophora cellulose composites prepared from a dispersion containing a $0.4 \mathrm{M}$ pyrrole solution (Clad_fibers-1), b $0.8 \mathrm{M}$ pyrrole solution (Clad_fibers-1double), and c $0.4 \mathrm{M}$ pyrrole solution and two consecutive polymerizations (Clad_fibers-2)

spherical PPy particles deposited on top of the cellulose fibers. It should, however, be noted that the micrographs only represent very small parts of the samples and that spherical particles could be seen to some extent in all three samples. Nevertheless, these micrographs do suggest that the composites synthesized with larger amounts of pyrrole (Clad_fibers-1double and Clad_fibers-2) contain more PPy and have slightly thicker fibers compared to the standard composite (Clad_fibers-1). This indicates that it is possible 

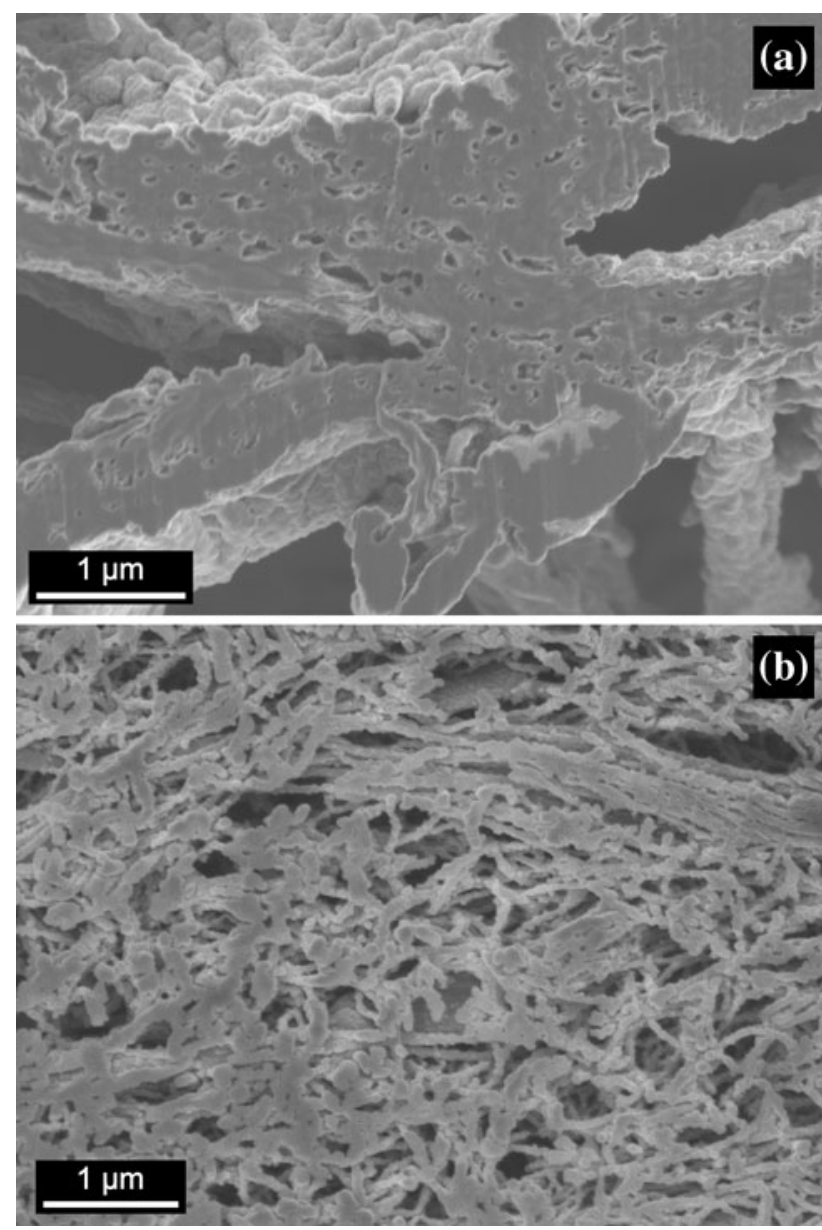

Fig. 3 Cross-section SEM micrographs of a a PPy/paper composite prepared by dip-coating of a filter paper sheet (Filter_paper_dip-1) and $\mathbf{b}$ a PPy/Cladophora cellulose composite prepared from a dispersion of Cladophora cellulose fibers

to increase the thickness of the PPy layer on the individual cellulose fibers to some extent.

To evaluate how the different obtained structures affect the electrochemical properties of the composites, cyclic voltammograms were recorded with the composites as working electrodes at a scan rate of $5 \mathrm{mV} / \mathrm{s}$ in $2.0 \mathrm{M} \mathrm{NaCl}$, as is shown in Fig. 4. It can be seen that all composites gave rise to well-defined voltammograms with oxidation peak potentials between $-0.25 \mathrm{~V}$ and $0.02 \mathrm{~V}$ vs. $\mathrm{Ag} / \mathrm{AgCl}$. The electrochemical behavior of the composites based on the dispersed Cladophora cellulose fibers are in good agreement with that found in previous reports $[12,22,23$, 32]. In Fig. 4, it can be seen that the magnitude of the currents (normalized with respect to the weight of the composite) increased significantly as a result of the repeated dip-coating process for the filter paper sheet composites, indicating an increased amount of PPy in the composites after each dip-coating step. For the dispersed Cladophora cellulose fiber composites, the effect of a repeated polymerization or increased amounts of reagents on the currents was, however, only marginal. It can also been seen that the normalized currents obtained with the composite prepared by dip-coating of Cladophora cellulose paper sheets were significantly smaller than those obtained with the composites prepared from dispersed Cladophora cellulose fibers. This demonstrates that polymerization on dispersed Cladophora cellulose fibers is a much more efficient way of making composite PPy based materials for energy storage applications than polymerization on Cladophora cellulose paper sheets. Another striking feature in the voltammograms in Fig. 4 is the considerably narrower oxidation peak for the composites prepared from the paper sheets than for the composites manufactured from dispersed cellulose fibers independent of the type of cellulose used. We ascribe this effect to differences in the electrochemical properties of the composites caused by the different polymerization conditions used for the dispersed fibers and paper sheets. In the dip-coating process pyrrole adsorbed on the paper sheets was subsequently exposed to the iron (III) chloride solution used as the oxidant while a mixture of pyrrole and iron (III) chloride was used with the dispersed fibers. The present results suggest that the former process results in PPy with a more narrow distribution of redox potentials [39] possibly due to differences in the polymerization process involving polymerization on the cellulose surface as opposed to predominantly in the solution as can be expected in the dispersed fiber process.

Cyclic voltammograms were also obtained for a range of additional scan rates up to $50 \mathrm{mV} / \mathrm{s}$. At scan rates higher than $50 \mathrm{mV} / \mathrm{s}$, the PPy oxidation peak was, however, found to overlap with the overoxidation [40] peak which rendered a separation of the charge contributions from these two processes impossible. The latter was mainly a result of an anodic shift in the PPy oxidation peak with increasing scan rate as the peak potential for the overoxidation peak remained practically independent of the scan rate. Since overoxidation leads to a degradation of the polymer [40], this process cannot be used for reversible charge storage and the charge contribution from the overoxidation reaction was therefore not included when evaluating the charge capacities of the composites from the voltammograms. In Fig. 5, the charge capacities per gram composite are shown as a function of the scan rate for four of the studied composites (Sample voltammograms are provided in the electronic supplementary material). Interestingly, there was no significant drop in the charge capacity for any of the samples. This suggests that the mass transport rates of the counter ions within the composite and the PPy layer were high enough to ensure a complete oxidation and reduction of the PPy layer at all the employed scan rates. This can be attributed to the porous nature of the composites seen in Figs. 1-3 and the presence of relatively thin PPy layers on 
Fig. 4 Cyclic voltammograms, recorded at $5 \mathrm{mV} / \mathrm{s}$ in $2.0 \mathrm{M}$ $\mathrm{NaCl}$, for the different PPy/ cellulose composites. The currents were normalized with respect to the composite weights
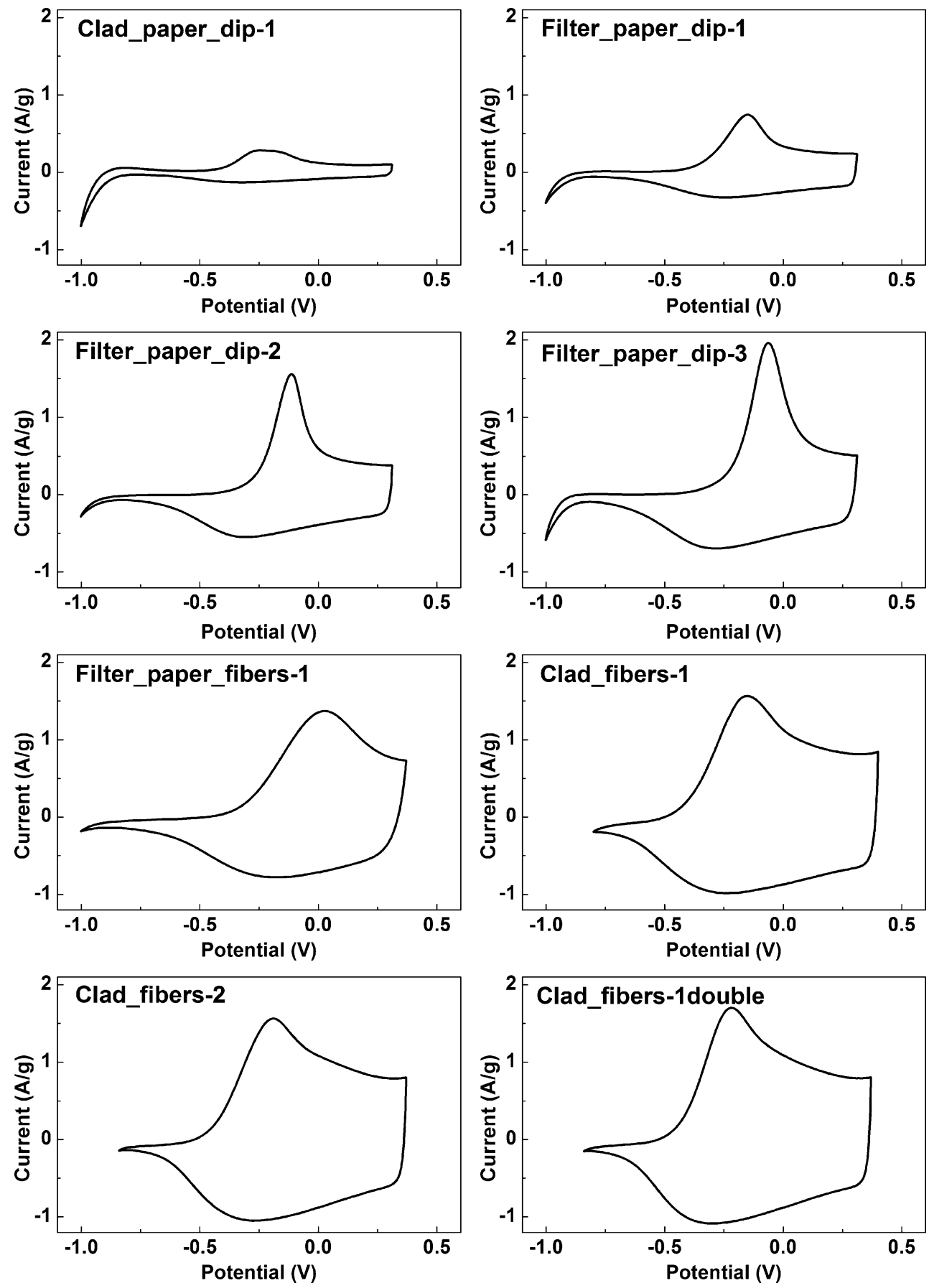

the individual cellulose fibers, at least for the Cladophora cellulose fibers. We have previously shown $[22,23]$ that the PPy layers are about $50 \mathrm{~nm}$ thick for the dispersed Cladophora cellulose fiber composites. In Fig. 5, it is clearly seen that the composite prepared from dispersed Cladophora cellulose fibers yielded the highest specific charge capacities (i.e., $\sim 150 \mathrm{C} / \mathrm{g}$ ) while the lowest specific charge capacity (i.e., $\sim 20 \mathrm{C} / \mathrm{g}$ ) was found for the dipcoated Cladophora cellulose paper sheet composite. The higher value of $150 \mathrm{C} / \mathrm{g}$ corresponds to an integrated specific capacitance of about $180 \mathrm{~F} / \mathrm{g}$ which agrees well with previously reported values for PPy in the literature [8]. The higher specific charge capacities for the dispersed Cladophora cellulose composites, when compared to the filter paper-based composites, are readily explained by the fact that the average Cladophora cellulose fiber diameter was significantly smaller than that for the wood based cellulose fibers present in the filter paper-based composites (see Fig. 1). The small specific capacity for the dip-coated Cladophora cellulose paper can, as will be discussed in 


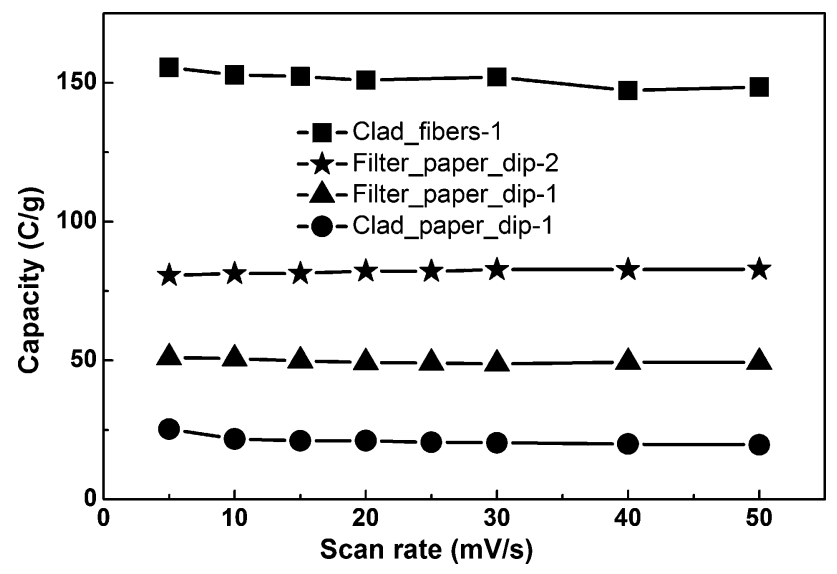

Fig. 5 Specific charge capacity as a function of the cyclic voltammetric scan rate, for the Clad_fibers-1 (squares), Filter_paper_dip-2 (stars), Filter_paper_dip-1 (triangles) and Clad_paper_dip-1 (circles) composites, respectively. The specific charge capacities, which were calculated based on the voltammograms, are given with respect to the weight of the composites

more detail below, be explained by the high crystallinity of the Cladophora cellulose fibers. In Fig. 5 it is likewise seen that the charge capacity of the dip-coated filter paper sheet composite could be increased from $\sim 50$ to $\sim 80 \mathrm{C} / \mathrm{g}$ by repeating the dip-coating process one time. These results, which confirm our previous conclusions based on the currents in the voltammograms in Fig. 4, clearly demonstrate the advantage of using the previously described polymerization process based on a dispersion of Cladophora cellulose fibers.

To investigate the cause of the higher specific charge capacities for the composite prepared from dispersed Cladophora cellulose fibers compared to those of the other composites, the specific charge capacity was plotted versus the amount of PPy in the different composites (the amounts were obtained by CHN-analysis). As is seen in Fig. 6, the specific charge capacity was found to depend rather linearly on the concentration of PPy in the composites indicating that the same fraction of the PPy layers (most likely close to $100 \%$ ) was electrochemically active in all composites. This is in good agreement with expectations based on previously published reports $[22,23]$.

As can be expected based on the results presented in Fig. 5, the highest PPy specific charge capacities (and PPy content) were found for the composites prepared from dispersed Cladophora cellulose fibers. For these composites, PPy contents up to $63 \mathrm{wt} \%$ were obtained whereas a content of only $12 \mathrm{wt} \%$, was reached for the composite prepared from a Cladophora cellulose paper sheet. The lower PPy concentration in the paper sheet composites compared to the composites prepared from dispersed fibers can be explained based on the dip-coating polymerization process because the yield of this procedure clearly is

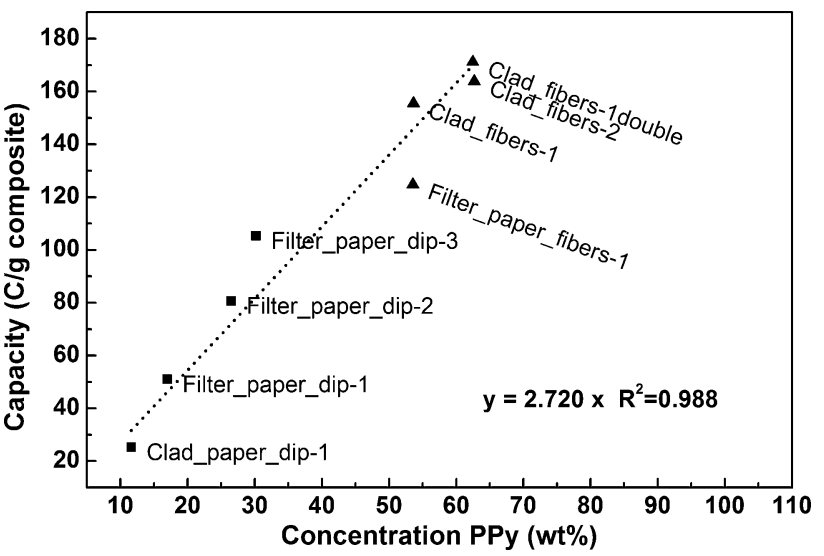

Fig. 6 Specific charge capacity (with respect to the weight of the composites) as a function of the PPy concentration in the composite. The composites prepared from paper sheets are represented by squares whereas the composites prepared from dispersed fibers are represented by triangles. The specific charge capacity was calculated based on the voltammetric oxidation peaks obtained at $5 \mathrm{mV} / \mathrm{s}$ while the PPy content was determined by elemental analysis of nitrogen

limited by the amount of pyrrole solution absorbed by the paper sheets. This also explains the observed increase in the charge storage capacity (and PPy content) as a result of a second and a third dip-coating step for the composites prepared using the filter paper sheets. For composites prepared from dispersed cellulose fibers, the PPy concentration is instead expected to be controlled by the amount of pyrrole present in the dispersion. When comparing the results for the three composites prepared from dispersed Cladophora cellulose fibers it is, however, evident that the use of double the amount of pyrrole and $\mathrm{FeCl}_{3}$ (see Fig 6, Clad_fibers-1double), or a repeated polymerization (see Fig. 6, Clad_fibers-2) only resulted in an $\sim 5 \%$ increase in the specific charge capacity (yielding 171 and $164 \mathrm{C} / \mathrm{g}$, respectively) compared to the standard dispersed Cladophora cellulose fiber composite (see Fig. 6, Clad_fibers-1). The standard Cladophora cellulose dispersed fiber composite contained $54 \mathrm{wt} \%$ PPy while the corresponding PPy concentrations in Clad_fibers-1double and Clad_fibers-2 were 62 and $63 \mathrm{wt} \%$, respectively. These results suggest that there is an upper limit for the PPy layer thickness on the dispersed Cladophora cellulose fibers that can be reached with the present polymerization procedure. The reasons for this are not yet fully understood but could involve adherence problems causing a loss of PPy during the composite washing step. This hypothesis is supported by the fact that the washing solution turned slightly black for the Clad_fibers-1double composite and the results of a study by Ichinose et al. [35], in which a QCM resonator was repeatedly immersed in the polymerization solution and insoluble polymer was observed to precipitate from the reaction mixture and attach to the QCM resonator. This 
polymer was, however, easily removed by washing, indicating poor polymer-polymer adhesion.

The significantly lower specific charge capacity (and PPy content) for the composite prepared from a Cladophora cellulose paper sheet (Clad_paper_dip-1) compared to the corresponding composite made using a filter paper sheet (Filter_paper_dip-1) may at first, seem surprising based on the morphological characterization of the two composites, which showed that the Cladophora cellulose paper had a high fiber surface area [38]. A possible explanation could, however, be that the Cladophora cellulose fibers are highly crystalline [38] which means that a solution, such as the aqueous pyrrole solution used in the present synthesis, cannot penetrate the fibers during the soaking stage of the synthesis but instead is restricted to the fiber surface and the interstitial space between the fibers [41, 42]. The hypothesis is further supported by the fact that the BET specific surface area for the Cladophora cellulose was of the same order of magnitude $\left(\sim 50-100 \mathrm{~m}^{2} / \mathrm{g}\right)$ with both nitrogen and water adsorption [38], proving that, in this material, water could not find additional attachment sites compared to those found by the non-reactive nitrogen molecules [41]. For the composites made with cellulose from wood fibers the situation is different. Such fibers have a significantly lower degrees of crystallinity [38, 43], and the specific surface area probed by water adsorption is significantly higher $\left(\sim 120-200 \mathrm{~m}^{2} / \mathrm{g}\right)$ than that probed by nitrogen $[38,44]\left(\sim 0.5-1 \mathrm{~m}^{2} / \mathrm{g}\right)$. Waterbased solutions can, therefore, easily penetrate these cellulose fibers. Celluloses with lower degrees of crystallinity also have a higher average number of hydrogen bonds between water molecule and cellulose, verifying the deep fiber penetration [45].

The fact that the specific surface area available for a water-based solution was higher for the wood cellulose (than for the Cladophora cellulose) also suggests that PPy could have been present both on the surface and within the wood fibers. PPy has, in fact, previously been found to be formed in the amorphous regions of cellulose fibers [46, 47]. Further support for the presence of PPy within the wood fibers can be found by scrutinizing the PPy concentration data in Fig. 6. Since the average diameter of the wood fibers was of the order of micrometers while that for the Cladophora fibers typically was $\sim 50 \mathrm{~nm}$, the presence of only a PPy film on the surface of the wood fibers would not give rise to the PPy concentrations seen in Fig. 6. To explain the PPy concentrations seen in Fig. 6, PPy must hence also have been present within the wood fibers. Although, it is possible that PPy to some extent was present within the Cladophora cellulose fibers, most of the PPy was undoubtedly present on the surface of these fibers due to the limited volume of the crystalline (and thus more water inaccessible) fibers. As is seen in Fig. 6, the PPy contents of the filter paper sheet composites were all relatively low (below $31 \mathrm{wt} \%$; cf. Fig. 6) in comparison with those for the composites made using the dispersed fiber approach. The higher PPy concentration for the dispersed fiber composites as compared with the corresponding dip-coated composites can be explained by the larger total fiber surface area exposed to the polymerization solution in the dispersed fiber approach. This effect should clearly facilitate the attainment of a more complete coverage of the cellulose fibers.

\section{Conclusions}

The specific charge capacities of cellulose-polypyrrole (PPy) composites, manufactured from commercial filter paper and Cladophora cellulose paper sheets, as well as dispersed wood and Cladophora cellulose fibers, were found to be proportional to the PPy content of the composite, despite differences in the polymerization procedure, and the highest specific charge capacity (i.e., $171 \mathrm{C} / \mathrm{g}$ ) was obtained for composites manufactured from dispersed Cladophora cellulose fibers. This charge capacity corresponds to $274 \mathrm{C} / \mathrm{g}$ when normalized with respect to the PPy content of the sample. Dip-coating of premade paper sheets was found to result in composites with lower specific charge capacities than when polymerization on dispersed fibers was carried out prior to the paper making step. SEM analyses showed that it is possible to form PPy throughout a sheet of filter paper using the straightforward dip-coating polymerization process and that the composites prepared from a dispersion of Cladophora cellulose fibers exhibited very similar structures in the bulk as on the surface. It is concluded that the Cladophora cellulose composite was composed of cellulose fibers coated with a thin layer of PPy whereas the PPy in the filter paper composites may also have been situated within the wood cellulose fibers. The latter can be explained by the higher accessibility of the aqueous pyrrole solution to wood-based fibers as compared to high crystallinity algae based cellulose fibers. The present results clearly show that the cellulose substrate and polymerization procedure are very important factors in the development of new paper-based electrode materials for inexpensive, flexible and environmentally friendly energy storage devices.

Acknowledgements Kjell Jansson at Stockholm University is gratefully acknowledged for his help with the cross-section micrographs. The authors acknowledge financial support from the European Institute of Innovation and Technology, under the KIC InnoEnergy NewMat and electrical energy storage projects, the Swedish Foundation for Strategic Research (SSF) (grant RMA08-0025), the Swedish Science Council (VR), the Bo Rydin Foundation, the Nordic Innovation Centre (contract number 10014) and SweGRIDS (Energimyndigheten contract number 35300-1).

Open Access This article is distributed under the terms of the Creative Commons Attribution License which permits any use, 
distribution, and reproduction in any medium, provided the original author(s) and the source are credited.

\section{References}

1. Nishide H, Oyaizu K (2008) Science 319(5864):737. doi:10.1126/ science. 1151831

2. Geng JQ, Bonnet JP, Renault S, Dolhem F, Poizot P (2010) Energy Environ Sci 3(12):1929. doi:10.1039/c0ee00126k

3. Tarascon JM, Armand M (2001) Nature 414(6861):359. doi: $10.1038 / 35104644$

4. Nam KT, Kim DW, Yoo PJ, Chiang CY, Meethong N, Hammond PT, Chiang YM, Belcher AM (2006) Science 312(5775):885. doi:10.1126/science.1122716

5. Rogers JA, Someya T, Huang YG (2010) Science 327(5973): 1603. doi:10.1126/science. 1182383

6. Scrosati B (2007) Nature Nanotech 2(10):598. doi:10.1038/ nnano. 2007.318

7. Hu LB, Choi JW, Yang Y, Jeong S, La Mantia F, Cui LF, Cui Y (2009) Proc Natl Acad Sci USA 106(51):21490. doi:10.1073/ pnas.0908858106

8. Snook GA, Kao P, Best AS (2011) J Power Sources 196(1):1. doi:10.1016/j.jpowsour.2010.06.084

9. Katz HE, Searson PC, Poehler TO (2010) J Mater Res 25(8):1561. doi:10.1557/jmr.2010.0201

10. Shirakawa H, Louis EJ, MacDiarmid AG, Chiang CK, Heeger AJ (1977) J Chem Soc Chem Commun 16:578. doi:10.1039/C3977 0000578

11. Novak P, Muller K, Santhanam KSV, Haas O (1997) Chem Rev 97(1):207. doi:10.1021/cr941181o

12. Nyholm L, Nyström G, Mihranyan A, Strømme M (2011) Adv Mater 23(33):3751. doi:10.1002/adma.201004134

13. Lota K, Khomenko V, Frackowiak E (2004) J Phys Chem Solids 65(2-3):295. doi:10.1016/j.jpcs.2003.10.051

14. Li CM, Sun CQ, Chen W, Pan L (2005) Surf Coat Technol 198(1-3):474. doi:10.1016/j.surfcoat.2004.10.065

15. Jurewicz K, Delpeux S, Bertagna V, Beguin F, Frackowiak E (2001) Chem Phys Lett 347(1-3):36. doi:10.1016/s0009-2614 (01)01037-5

16. Sasso C, Zeno E, Petit-Conil M, Chaussy D, Belgacem MN, Tapin-Lingua S, Beneventi D (2010) Macromol Mater Eng 295:934. doi:10.1002/mame.201000148

17. Huang J, Ichinose I, Kunitake T (2005) Chem Commun 13:1717. doi:10.1039/b415339a

18. Johnston JH, Kelly FM, Moraes J, Borrmann T, Flynn D (2006) Curr Appl Phys 6(3):587. doi:10.1016/j.cap.2005.11.067

19. Inzelt G (2008) Conducting polymers: a new era in electrochemistry. Springer, Berlin

20. Wang JZ, Chou SL, Liu H, Wang GX, Zhong C, Chew SY, Liu HK (2009) Mater Lett 63(27):2352. doi:10.1016/j.matlet.2009. 08.007

21. Liu R, Cho S II, Lee SB (2008) Nanotechnology 19(21):215710. doi:10.1088/0957-4484/19/21/215710

22. Mihranyan A, Nyholm L, Garcia Bennett AE, Strømme M (2008) J Phys Chem B 112(39):12249. doi:10.1021/jp805123w
23. Nyström G, Razaq A, Strømme M, Nyholm L, Mihranyan A (2009) Nano Lett 9(10):3635. doi:10.1021/n1901852h

24. Razaq A, Nyholm L, Sjödin M, Strømme M, Mihranyan A (2012) Adv Energy Mater. doi:10.1002/aenm.201100713

25. Gelin K, Mihranyan A, Razaq A, Nyholm L, Strømme M (2009) Electrochim Acta 54(12):3394. doi:10.1016/j.electacta.2009.01.010

26. Razaq A, Mihranyan A, Welch K, Nyholm L, Strømme M (2009) J Phys Chem B 113(2):426. doi:10.1021/jp806517h

27. Strømme M, Frenning G, Razaq A, Gelin K, Nyholm L, Mihranyan A (2009) J Phys Chem B 113(14):4582. doi:10.1021/jp9002627

28. Razaq A, Nyström G, Strømme M, Mihranyan A, Nyholm L (2011) PLoS One 6(12):e29243. doi:10.1371/journal.pone. 0029243

29. Ferraz N, Carlsson DO, Hong J, Larsson R, Fellström B, Nyholm L, Strømme M, Mihranyan A (2012) J R Soc Interface. doi:10.1098/ rsif.2012.0019

30. Ferraz N, Strømme M, Fellström B, Pradhan S, Nyholm L, Mihranyan A (2012) J Biomed Mater Res Part A. doi:10.1002/ jbm.a.34070

31. Nyström G, Mihranyan A, Razaq A, Lindström T, Nyholm L, Strømme M (2010) J Phys Chem B 114(12):4178. doi:10.1021/ jp911272m

32. Olsson H, Nyström G, Strømme M, Sjödin M, Nyholm L (2011) Electrochem Commun 13(8):869. doi:10.1016/j.elecom.2011.05.024

33. Björklund RB, Lundström I (1984) J Electron Mater 13(1):211. doi:10.1007/BF02659844

34. Winther-Jensen B, Clark N, Subramanian P, Helmer R, Ashraf S, Wallace G, Spiccia L, MacFarlane DR (2007) J Appl Polym Sci 104(6):3938. doi:10.1002/app.26162

35. Ichinose I, Kunitake T (1999) Adv Mater 11(5):413. doi:10.1002/ (sici)1521-4095(199903)11:5<413:aid-adma413>3.0.co;2-d

36. Grunze M, Lamb RN (1987) Chem Phys Lett 133(4):283. doi:10.1016/0009-2614(87)87068-9

37. Pääkkö M, Vapaavuori J, Silvennoinen R, Kosonen H, Ankerfors M, Lindström T, Berglund LA, Ikkala O (2008) Soft Matter 4(12):2492. doi:10.1039/b810371b

38. Mihranyan A, Llagostera AP, Karmhag R, Strømme M, Ek R (2004) Int J Pharm 269(2):433. doi:10.1016/j.ijpharm.2003. 09.030

39. Heinze J, Frontana-Uribe BA, Ludwigs S (2010) Chem Rev 110(8):4724. doi:10.1021/cr900226k

40. Pud AA (1994) Synth Met 66(1):1. doi:10.1016/0379-6779(94) 90155-4

41. Strømme M, Mihranyan A, Ek R, Niklasson GA (2003) J Phys Chem B 107(51):14378. doi:10.1021/jp034117w

42. Mihranyan A, Strømme M (2004) Chem Phys Lett 393(4-6):389. doi:10.1016/j.cplett.2004.06.043

43. Park S, Johnson DK, Ishizawa CI, Parilla PA, Davis MF (2009) Cellulose 16(4):641. doi:10.1007/s10570-009-9321-1

44. Mihranyan A, Strømme M, Ek R (2006) Eur J Pharm Sci 27(2-3):220. doi:10.1016/j.ejps.2005.10.002

45. Heidarian M, Mihranyan A, Strømme M, Ek R (2006) Int J Pharm 323(1-2):139. doi:10.1016/j.ijpharm.2006.05.058

46. Dall'Acqua L, Tonin C, Peila R, Ferrero F, Catellani M (2004) Synth Met 146(2):213. doi:10.1016/j.synthmet.2004.07.005

47. Dall'Acqua L, Tonin C, Varesano A, Canetti M, Porzio W, Catellani M (2006) Synth Met 156(5-6):379. doi:10.1016/ j.synthmet.2005.12.021 